\title{
Centrifugal Distortions in Molecules: An Ab Initio Approach
}

\section{with Application to Ozone}

\author{
Lawrence L. Lohr and A. J. Helman \\ Department of Chemistry, University of Michigan, Ann Arbor, Michigan 48109
}

Received 2 June 1986; accepted 1 October 1986

\begin{abstract}
Our procedure for employing analytical gradients of $a b$ initio potential energy hypersurfaces in the description of centrifugally distorted molecules is applied for the first time to an asymmetric top, namely ozone. Both single determinantal (HF/6-31G*) and analytically fitted multiconfigurational self-consistent field surfaces were utilized. The focus of the $\mathrm{HF} / 6-31 \mathrm{G} *$ study is upon the centrifugal distortion pathway. Quartic centrifugal spectroscopic coefficients are obtained in both cases and are in reasonable agreement with experiment.
\end{abstract}

\section{INTRODUCTION}

In a recent publication ${ }^{1}$ we outlined a procedure for employing analytical gradients of $a b$ initio potential energy hypersurfaces in the description of centrifugally distorted molecules. Stationary points were located on the effective hypersurface defined as the sum of electronic and rotational energies. Centrifugal distortion pathways and centrifugal stabilization energies were defined; from the latter quartic centrifugal distortion spectroscopic constants were obtained for $\mathrm{H}_{2}^{+}, \mathrm{NH}_{3}, \mathrm{CH}_{4}, \mathrm{BF}_{3}$, and $\mathrm{SF}_{6}$. Comparisons with experimentally determined constants were generally quite satisfactory, with calculated values being typically $10 \%$ smaller than those observed and with this error being primarily the result of the overestimation of the curvature of the electronic hypersurfaces at the computational level employed, namely HF/6-31G**. Particularly satisfying was the excellent description of both the scalar and tensor quartic coefficients, $D_{s}$ and $D_{t}$, respectively, for the spherical tops $\mathrm{CH}_{4}$ and $\mathrm{SF}_{6}$. In the present article we outline an extension of the method to asymmetric tops and give results for a particularly important molecule, namely ozone, for which we have also made a theoretical study of the effects of anharmonicity on its ultraviolet continuum band shape. ${ }^{2}$

\section{METHODS}

We define an effective potential energy hypersurface $E(\mathbf{Q}, J)$ as

$$
E(\mathbf{Q}, \mathbf{J})=E_{\mathrm{el}}(\mathbf{Q})+E_{r}(\mathbf{Q}, \mathbf{J})
$$

where $E_{\text {el }}$ denotes the electronic energy, $E_{r}$ the rotational energy, $\mathbf{Q}$ the set of nuclear coordinates, and $J$ the rotational angular momentum. Molecular vibration is ignored in the present form of our method, while molecular rotation is treated classically. Thus the method provides a description of vibrational ground states in terms of vibrationless rotating deformable bodies.

We locate stationary points on the hypersurface by the condition that $\nabla E(\mathbf{Q}, J)=0$. Such points are not necessarily local minima as they may be saddle points or local maxima instead. However, for structures close to the true $(\mathbf{J}=0)$ equilibrium geometry they have typically been found ${ }^{1}$ to be local minima. In most of the examples considered in our previous study ${ }^{1}$ the use of cylindrical coordinates led to a rotational energy $E_{r}$ which depended upon only one coordinate. Thus for a total of $n=3 N-6$ internal coordinates, where $N$ is the number of atoms in the molecule, there were $n$ equations to be solved, namely

$$
\partial E_{\mathrm{el}} / \partial Q_{i}=0, \quad i=1, \quad n-1
$$




$$
\partial\left(E_{\mathrm{el}}+E_{r}\right) / \partial Q_{n}=0
$$

where $Q_{n}$ is the single coordinate appearing in $E_{r}$. One exception was our treatment of the rotation of $\mathrm{NH}_{3}$ about an axis perpendicular to the $\mathrm{C}_{3}$ axis. In this case the rotational energy depended upon three coordinates, so that three equations of the type of $(2 \mathrm{~b})$ had to be solved simultaneously.

Selecting a principal axis system to describe the rotations of an asymmetric top, we write $E_{r}$ simply as

$$
\begin{aligned}
E_{r}(\mathbf{Q}, \mathbf{J})= & A(\mathbf{Q}, \mathbf{J}) J_{a}^{2}+B(\mathbf{Q}, \mathbf{J}) J_{b}^{2} \\
& +C(\mathbf{Q}, \mathbf{J}) J_{\mathrm{c}}^{2}
\end{aligned}
$$

where $J_{a}, J_{b}$, and $J_{c}$ are the projections of the rotational angular momentum on the axes $a, b$, and $c$. These axes are typically taken to correspond to the customary ordering of rotational constants as $A>B>C$, where these effective constants may depend on $J$ as well as $\mathbf{Q}$. For ozone $a$ is the in-plane axis perpendicular to the $\mathrm{C}_{2}$ axis, $b$ is the $\mathrm{C}_{2}$ axis, and $c$ is the axis perpendicular to the molecular plane. For asymmetric tops whose principal axis directions change with $\mathbf{J}$ it may be more convenient not to assume a principal axis representation to begin with, but for ozone this is not the situation. The distortions accompanying rotation about any one of the principal axes of ozone preserve the $\mathrm{C}_{2 v}$ symmetry, so that there are only two structural parameters. We find it convenient to describe the molecule using the Cartesian coordinates $(x, y, z)$ for the three atoms as $(2 d, O, O),(-d, O, e)$ and $(-d, O,-e)$. These coordinates may be viewed as cylindrical coordinates for $\mathbf{J} \| x$ and $\mathbf{J} \| z$, but not for $\mathbf{J} \| y$. The Cartesian axes are associated with the rotational axes according to the $I^{r}$ representation, namely $x$ with $b, y$ with $c$, and $z$ with $a$, this association being particularly desirable for an asymmetric top such as ozone which is close to being a prolate symmetric top. In terms of the distances $d$ and $e$ the bond length $R$ and bond angle $\theta$ are given by

$$
\begin{aligned}
R & =\left(9 d^{2}+e^{2}\right)^{1 / 2} \\
\theta & =\cos ^{-1}\left[\left(R^{2}-2 e^{2}\right) / R^{2}\right]
\end{aligned}
$$

The moments of inertia are

$$
\begin{aligned}
& I_{a}=6 m_{\mathrm{o}} d^{2} \\
& I_{b}=2 m_{\mathrm{o}} e^{2} \\
& I_{c}=I_{\mathrm{a}}+I_{\mathrm{b}}
\end{aligned}
$$

where $m_{0}$ is mass of an $\mathrm{O}$ atom.
The condition $\nabla E(\mathbf{Q}, \mathbf{J})=0$ when combined with the moments of inertia in (5) yield the following:

$$
\begin{aligned}
& \mathbf{J} \| a \\
& \quad \partial E_{\mathrm{el}} / \partial d-J_{a}^{2} / 6 m_{\mathrm{o}} d^{3}=0 \\
& \quad \partial E_{\mathrm{el}} / \partial e=0 \\
& \mathbf{J} \| b \\
& \quad \partial E_{\mathrm{el}} / \partial d=0 \\
& \quad \partial E_{\mathrm{el}} / \partial e-J_{b}^{2} / 2 m_{\mathrm{o}} e^{3}=0 \\
& \mathbf{J} \| c \\
& \partial E_{\mathrm{el}} / \partial d-3 d J_{c}^{2} / 2 m_{0}\left(3 d^{2}+e^{2}\right)^{2}=0 \\
& \partial E_{\mathrm{el}} / \partial e-e J_{c}^{2} / 2 m_{\mathrm{o}}\left(3 d^{2}+e^{2}\right)^{2}=0
\end{aligned}
$$

For $\mathbf{J} \| a$ we simply select $d$, obtain $e$ by criterion $(6 \mathrm{~b})$, calculate $\partial E_{\mathrm{el}} / \partial d$ at the structure corresponding to $d$ and $e$, and then solve (6a) for $J_{a}$. The procedure for $J \| b$ is similar, while that for $\mathbf{J} \| c$ requires a simultaneous solution of (6e) and (6f) which may be expressed by the condition that

$$
\left(\partial E_{e l} / \partial d\right) /\left(\partial E_{\mathrm{el}} / \partial e\right)=3 d / e
$$

We define as before ${ }^{1}$ a centrifugal stabilization energy $\Delta E$ as the difference between the energy of a rigid molecule with $J$ and that of the deformable molecule with the same $\mathbf{J}$. That is,

$$
\Delta E(\mathbf{J})=\Delta E_{\mathrm{el}}(\mathbf{J})+\Delta E_{r}(\mathbf{J})
$$

where $\Delta E_{\mathrm{el}}(J)$ is the negative difference between $E_{\text {el }}$ at $\mathbf{Q}=\mathbf{Q}^{\circ}$, the equilibrium geometry, and at $\mathbf{Q}=\mathbf{Q}(\mathbf{J})$, the quasiequilibrium geometry for a given $J$, and $\Delta E_{r}$ is the positive difference between $E_{r}$ at $\mathbf{Q}=\mathbf{Q}^{\circ}$ and at $\mathbf{Q}=\mathbf{Q}(\mathbf{J})$. Typically $\Delta E_{r}$ has approximately twice the magnitude of $\Delta E_{\mathrm{el}}$, so that the sum $\Delta E$ is positive with one-half the magnitude of $\Delta E_{r}$.

It may appear inconsistent to assume a classical description of molecular rotation for an asymmetric top and then to consider in (6c) and (6d) $J$ to be parallel to the intermediate principal axis $b$, as such a rotation is unstable in the sense that it corresponds to a saddle point on the rotational energy surface, with intersecting contours of constant energy, corresponding to semiclassical trajectories, leading away from it. However, 
the Eqs. (6c) and (6d) representing the quasiequilibrium are nonetheless valid, as $\mathbf{J}$ parallel to any principal axis implies dynamical balance, albeit unstable in some cases, with $\mathbf{J}$ parallel to the angular velocity $\omega$. What we are so far unable to describe with our method are the dynamically unbalanced cases of $\boldsymbol{J}$ not parallel to a principal axis and hence not parallel to $\omega$.

The electronic structure calculations were made at two levels, the first being the singledeterminantal level using the GAUSSIAN 80 and 82 programs ${ }^{3}$ with the split valence plus polarization basis set $6-31 \mathrm{G}^{*}{ }^{4,5}$ This computational level is designated $\mathrm{HF} / 6-31 \mathrm{G}^{*}$. Analytical gradients were employed in locating both the unconstrained $(J=0)$ and constrained $(J \neq 0)$ stationary points as described below. Analytical second derivatives were employed in calculating vibrational frequencies, although the latter are not used in our procedure for obtaining centrifugal distortion constants.

The second level of electronic structure calculations employed a published analytical $\mathrm{fit}^{6}$ by Sheppard and Walker of the generalized valence bond perfect pairing (GVB-PP) multiconfiguration self-consistent field (MCSCF) hypersurface calculated ${ }^{7}$ by Hay et al. This excellent surface was also used as the ground state in our recent theoretical study of the ultraviolet continuum band shape. $^{2}$

\section{RESULTS}

In Table I we present the basic computed parameters bond length $R$, bond angle $\theta$, rotational constants $A, B$, and $C$ and vibrational frequencies $\bar{\nu}_{1}, \bar{\nu}_{2}$, and $\bar{\nu}_{3}$. As expected, ${ }^{8,9}$ the $\mathrm{HF} / 6-31 \mathrm{G}^{*}$ level yields a computed bond length which is too short ${ }^{10}$ and vibrational frequencies which are too large. ${ }^{11}$ However, these errors will tend to cancel in the calculation of centrifugal distortion constants. The results obtained for these parameters by using the SheppardWalker (SW) fit ${ }^{6}$ of the GVB-PP MCSCF surface ${ }^{7}$ are very much better. The vibrational frequencies were obtained by us using analytical second derivatives of the sw surface and differ somewhat from those reported by Hay et al. ${ }^{7}$

As in our previous study ${ }^{1}$ the $\mathrm{HF} / 6-31 \mathrm{G}^{*}$ level is used to explore energies and distortions for large $J$ values, with information for low $J$ obtained by extrapolation. In Figure 1 we show the centrifugal stabilizations in the form of $\Delta E / J^{4} v s J$ using $\Delta E$ from (8) for the three cases $\mathbf{J}\|a, \mathbf{J}\| b$, and $\mathbf{J} \| c$. From the extrapolations to $J=0$ shown by the dashed lines we obtain the quartic coefficients $D_{a}$, $D_{b}$, and $D_{c}$ as listed in Table I. The relationship of these parameters to parameters $\Delta_{J}$, $\Delta_{J K}, \Delta_{K}, \delta_{J}$, and $\delta_{K}$ is also given in Table I. The latter parameters appear as coefficients in the quartic portion of the quantum mechanical rotational hamiltonian ${ }^{12}$ as follows

Table I. Computed molecular parameters ${ }^{a}$ for $\mathrm{O}_{3}$.

\begin{tabular}{lccc}
\hline & \multicolumn{2}{c}{ Calculated } & \\
\cline { 2 - 3 } \multicolumn{1}{c}{ Parameter } & HF $^{\mathrm{b}}$ & MCSCF $^{\mathrm{e}}$ & Observed \\
\hline$R$ & 1.204 & 1.304 & $1.278^{\mathrm{d}}$ \\
$\theta$ & 119.0 & 115.8 & $116.8^{\mathrm{d}}$ \\
$A$ & 4.233 & 3.2885 & $3.55366633^{\mathrm{e}}$ \\
$B$ & 0.489 & 0.4322 & $0.445283212^{\mathrm{e}}$ \\
$C$ & 0.439 & 0.3822 & $0.394751810^{\mathrm{e}}$ \\
$\bar{\nu}_{1}\left(a_{1}\right)$ & 1537.5 & 1209.0 & $1134.9^{\mathrm{f}}$ \\
$\bar{\nu}_{2}\left(a_{1}\right)$ & 849.3 & 680.7 & $716.0^{\mathrm{f}}$ \\
$\bar{\nu}_{3}\left(b_{2}\right)$ & 1454.1 & 1086.1 & $1089.2^{\mathrm{f}}$ \\
$D_{a}=\Delta_{J}+\Delta_{J K}+\Delta_{K}$ & $2.55 \times 10^{-4}$ & $1.75 \times 10^{-4}$ & $2.102694325 \times 10^{-4 \mathrm{e}}$ \\
$D_{b}=\Delta_{J}+2 \delta_{J}$ & $4.85 \times 10^{-7}$ & $7.25 \times 10^{-7}$ & $5.93864892 \times 10^{-7 \mathrm{e}}$ \\
$D_{c}=\Delta_{J}-2 \delta_{J}$ & $2.48 \times 10^{-7}$ & $4.59 \times 10^{-7}$ & $3.1467846 \times 10^{-7 \mathrm{e}}$ \\
\hline
\end{tabular}

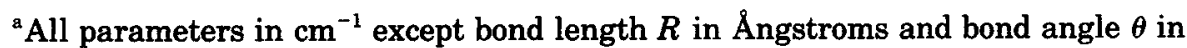
degrees.

${ }^{\mathrm{b}} \mathrm{HF} / 6-31 \mathrm{G} *$ level.

'Analytically fitted GVB.PP MCSCF level (Refs. 6 and 7).

${ }^{\mathrm{d}}$ Reference 10.

'Reference 12.

${ }^{f}$ Reference 11; harmonic frequencies, not fundamentals, are tabulated. 

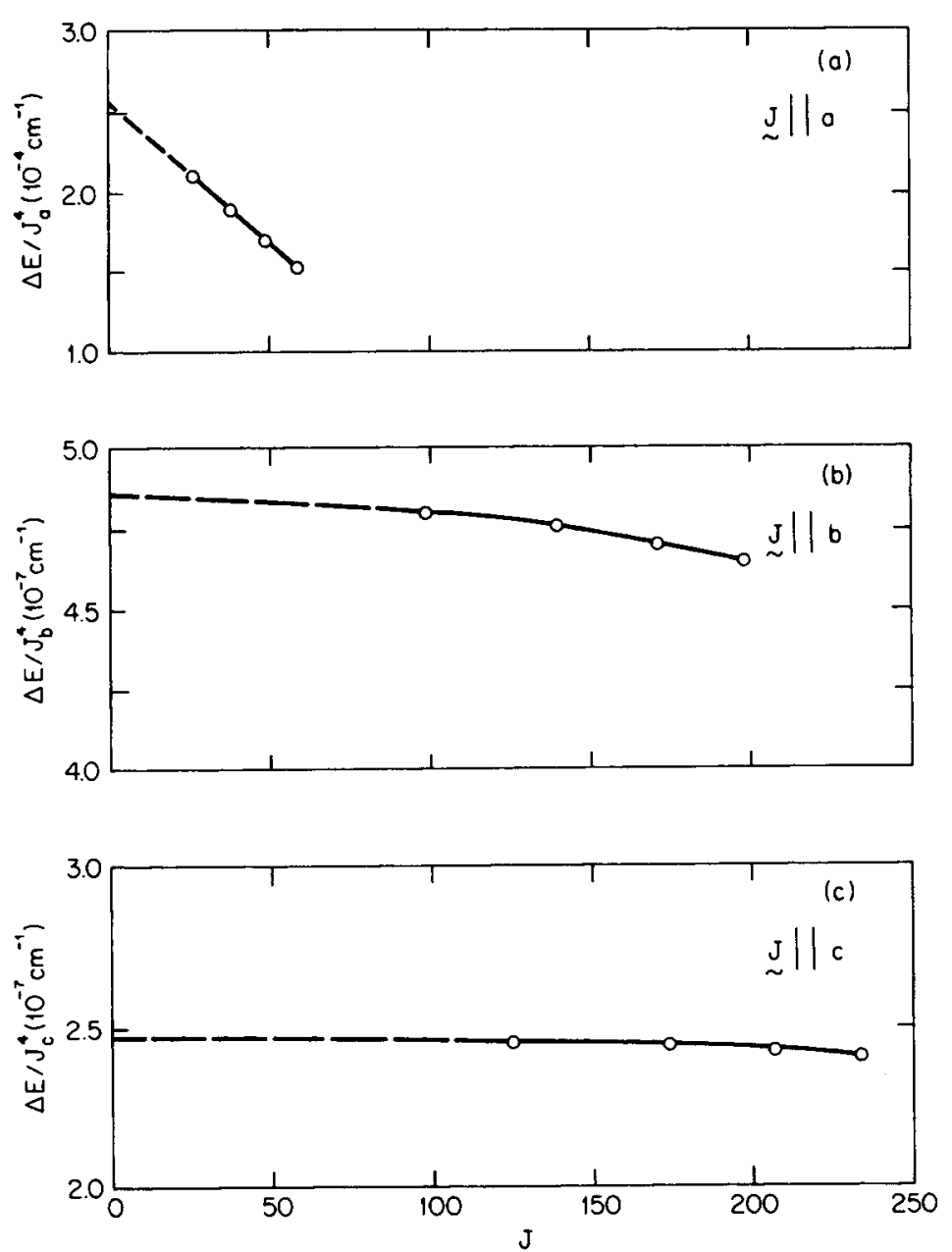

Figure 1. Centrifugal stabilizations $\Delta E / J^{4}$ in $\mathrm{cm}^{-1}$ vs. $J$ for $\mathrm{O}_{3}$ at the HF/6-31G* level for (a) $\mathbf{J} \| a$, (b) $\mathbf{J} \| b$, and (c) $\mathbf{J} \| c$. Circles denote the computed points with the dashed lines giving the extrapolations to $J=0$. The associated $J$ values may be taken from the legend for Figure 2. $\Delta E$ is defined in Eq. (8).

$$
\begin{aligned}
H_{4}= & -\Delta_{J} J^{4}-\Delta_{J K} J^{2} J_{z}^{2} \\
& -\Delta_{K} J_{z}^{4}-2 \delta_{J} J_{x y}^{2} J^{2} \\
& -\delta_{K}\left(J_{z}^{2} J_{x y}^{2}+J_{x y}^{2} J_{z}^{2}\right)
\end{aligned}
$$

where $J^{2}=\mathbf{J} \cdot \mathbf{J}, J_{x y}^{2}=J_{x}^{2}-J_{y}^{2}$, and the $x$, $y$, and $z$ axes correspond to $b, c$, and $a$, respectively. The agreement with experiment ${ }^{12}$ is generally satisfactory even at the HF/6$31 G^{*}$ level. For the MCSCF level we have utilized our analytical expressions ${ }^{2}$ for the second derivatives to obtain directly the quartic distortion coefficients in the low $J$ limit, thus eliminating the need for extrapolations. The centrifugal distortion of ozone has been previously discussed ${ }^{13,14}$ in terms of the conventional description ${ }^{15}$ involving rotational constants, vibrational frequencies, and Coriolis coupling coefficients. Our method, as illustrated by the $\mathrm{HF} / 6-31 \mathrm{G} *$ results in Figure 1, involves a direct estimation of the total (electronic plus rotational) energy of a rotating molecule.
In Figure 2 we show the computed centrifugal distortion pathways as bond angle changes versus bond length changes for the three cases $\mathbf{J}\|a, \mathbf{J}\| b$, and $\mathbf{J} \| c$, again for the HF/6-31G* level. The results may be expressed in terms of $\mathbf{J}$ as follows
(a) $\mathbf{J} \| a$
$\Delta R=6.8 \times 10^{-6} J_{a}^{2}$ $\Delta \theta=-3.0 \times 10^{-3} J_{a}^{2}$
(b) $\mathbf{J} \| b$
$\Delta R=4.9 \times 10^{-7} J_{b}^{2}$ $\Delta \theta=1.1 \times 10^{-4} J_{b}^{2}$
(c) $\mathbf{J} \| c$
$\Delta R=4.8 \times 10^{-7} J_{c}^{2}$
$\Delta \theta=5.9 \times 10^{-5} J_{c}^{2}$

In the above $\Delta R$ is the change in bond length in Angstroms. $\Delta \theta$ is the change in bond angle in degrees, and $J_{a}, J_{b}$, and $J_{c}$ are the dimensionless components of $\mathbf{J}$. The displacements may be expressed in terms of the zero-order rotational energies using the calculated rotational constants from Table I as 
follows
(a) $\mathbf{J} \| a$
$\Delta R=1.6 \times 10^{-6} E_{a}^{0}$
$\Delta \theta=-7.1 \times 10^{-4} E_{\mathrm{a}}^{0}$
(b) $\quad \mathbf{J} \| b \quad \Delta R=1.0 \times 10^{-6} E_{b}^{0}$

$$
\Delta \theta=2.2 \times 10^{-4} E_{b}^{0}
$$
(c) $\mathbf{J} \| c \quad \Delta R=1.1 \times 10^{-6} E_{c}^{0}$

$$
\Delta \theta=1.3 \times 10^{-4} E_{c}^{0}
$$

In the above the energy components are in $\mathrm{cm}^{-1}$ while $\Delta R$ and $\Delta \theta$ are in Angstroms and degrees as before. We note that the bond lengthening is nearly isotropic when expressed in terms of rotational energy rather than angular momentum.

\section{SUMMARY}

We have reviewed the procedure for calculating centrifugal distortions and accompa- nying energy stabilizations from gradients of $a b$ initio potential energy hypersurfaces and applied the procedure for the first time to an asymmetric top, namely ozone. The resulting quartic centrifugal coefficients are in good agreement with experiment. The bond length change is found to be nearly isotropic when expressed in terms of rotational energy rather than in terms of angular momentum. We conclude that our quasistatic procedure ${ }^{1}$ provides a generally adequate picture of centrifugal distortions in ozone. The strength of our method lies in its use to answer the following question: What is the geometry and energy of a molecule with a large rotational angular momentum?

The authors wish to thank Dr. H.M. Pickett for helpful discussions concerning ozone and the University of Michigan Computing Center for the use of its facilities.
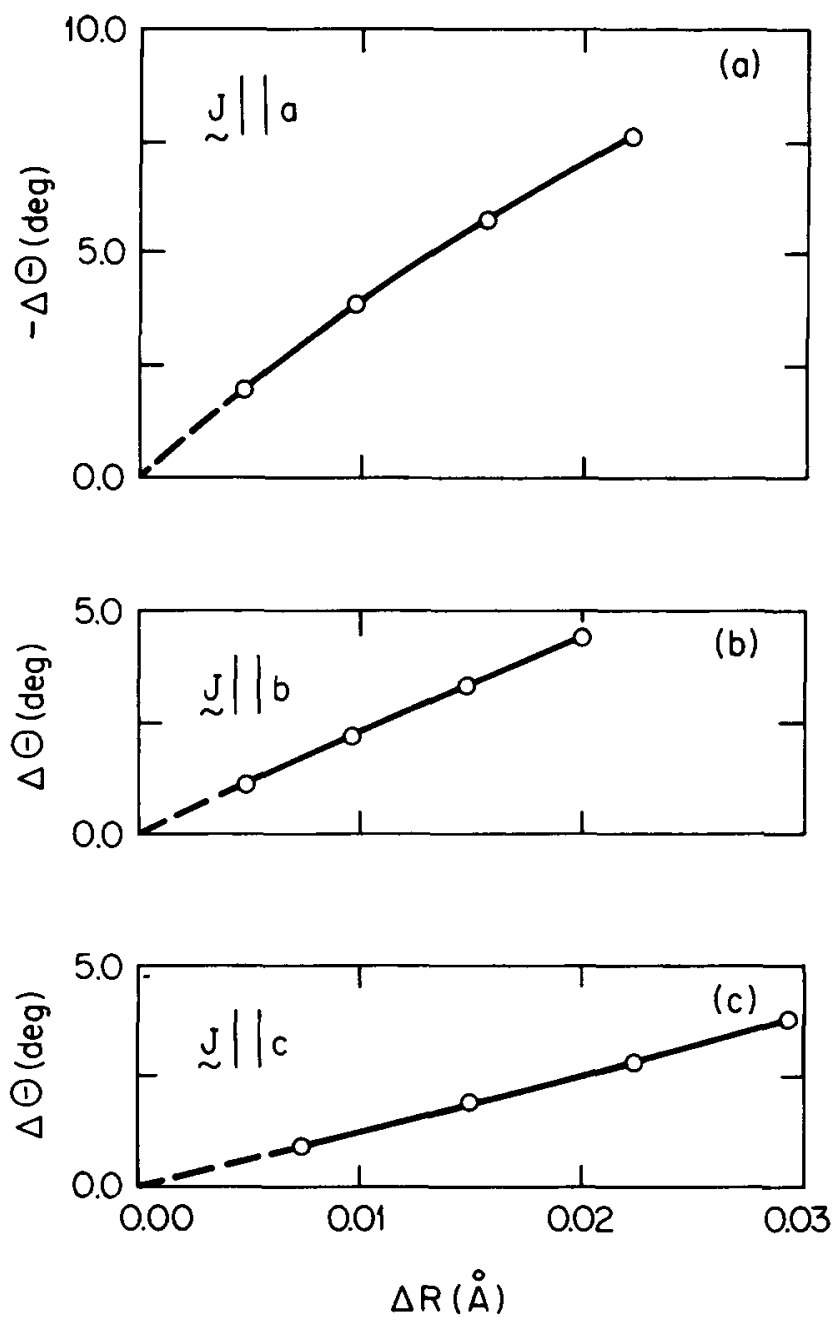

Figure 2. Centrifugal distortion pathways as bond angle changes $\Delta \theta$ in degrees versus bond length changes $\Delta R$ in Angstroms for $\mathrm{O}_{3}$ at the HF/6-31G* level for (a) $\mathbf{J} \| a$, (b) $\mathbf{J} \| b$, and (c) $\mathbf{J} \| c$. 


\section{References}

1. L. L. Lohr and J. -M. J. Popa, J. Chem. Phys., 84, 4196 (1986).

2. L. L. Lohr and A.J. Helman, J. Chem. Phys., in press.

3. J.S. Binkley, R.A. Whiteside, R. Krishnan, R. Seeger, D. J. DeFrees, H. B. Schlegel, S. Topiol, L. R. Kahn, and J. A. Pople, QCPE, 13, 406 (1980).

4. W. J. Hehre, R. Ditchfield, and J.A. Pople, J. Chem. Phys., 56, 2257 (1972).

5. R. C. Hariharan and J.A. Pople, Theor. Chim. Acta, 28, 213 (1973).

6. M. G. Sheppard and R. B. Walker, J. Chem. Phys., 78, 7191 (1983). Specifically the $a b$ initio, not the empirically adapted, fit was used in our study.

7. P. J. Hay, R. T. Pack, R. B. Walker, and E. J. Heller, J. Phys. Chem., 86, 862 (1982).

8. D.J. Frees, J.S. Binkley, and A.D. McLean, J. Chem. Phys., 80, 3720 (1984).

9. (a) J. A. Pople, H. B. Schlegel, R. Krishnan, D.J.
DeFrees, J.S. Binkley, M.J. Frisch, R.A. Whiteside, R. J. Hout, and W.J. Hehre, Int. J. Quantum Chem., Quantum Chem. Symp., 15, 269 (1981). (b) J.S. Binkley, M.J. DeFrees, R. Krishnan, R. A. Whiteside, B.S. Schlegel, E.M. Fluder, and J.A. Pople, Gaussian 82 Carnegie-Mellon University, Pittsburgh, PA, 1983.

10. T. Tanaka and Y. Morino, J. Mol. Spectros., 33, 538 (1970).

11. N. Monnanteuil, J.C. Depannemaeker, J. Bellet, A. Barbe, C. Secroun, P. Jouve, S. Giorgianni, Y. -S. Hoh, and K. N. Rao, J. Mol. Spectros., 71, 399 (1978).

12. A. Goldman, J.R. Gillis, D. G. Murcray, A. Barbe, and C. Secroun, J. Mol. Spectros., 96, 279 (1982).

13. J.C. Depannemaeker, B. Duterage, and J. Bellet, Compt. R. Acad. Sci. B., 279, 287 (1974).

14. A. G. Maki, J. Mol. Spectros., 57, 416 (1975).

15. J.K.G. Watson, in Vibrational Spectra and Structure, Vol. 6, J. R. Durig, Ed., Elsevier, Amsterdam, the Netherlands, 1977, pp. 2-89. 